\title{
A case of presumed acute retinal necrosis after intraocular foreign body injury
}

This article was published in the following Dove Press journal:

Clinical Ophthalmology

19 March 2013

Number of times this article has been viewed

\section{Sung Who Park' \\ Ik Soo Byon' \\ Hyun Jun Park ${ }^{2}$ \\ Ji Eun Lee ${ }^{1,3}$ \\ Boo Sup Oum ${ }^{1,3}$}

'Department of Ophthalmology, School of Medicine, Pusan National University, Busan, Korea; ${ }^{2}$ Department of Ophthalmology, Yangsan Pusan National University Hospital, Busan, Korea; ${ }^{3}$ Medical Research Institute, School of Medicine, Pusan National University Hospital, Busan, Korea
Correspondence: Ji Eun Lee

Department of Ophthalmology,

Pusan National University Hospital,

179 Gudeok-ro, Seo-gu, Busan, Korea

$\mathrm{Tel}+825$ I 2407957

Fax +825 I 242734

Email jlee@pusan.ac.kr
Abstract: The aim of this study was to report a case of acute retinal necrosis (ARN) after intraocular foreign body removal. A 32-year-old male presented with visual loss in the left eye. He was hit by an iron fragment while he was hammering. An intraocular foreign body was found with corneal laceration and traumatic cataract. On the day he was injured, primary closure of the laceration, lensectomy, and vitrectomy were performed, and the foreign body was removed. The day after the operation, there was no sign of retinal detachment or retinitis. Two days after the operation, retinal necrosis and accompanying vitreous inflammation were noted in the far periphery. On day 3, the necrosis spread circumferentially and inflammation became more distinct. ARN was presumed and intravenous acyclovir was administered. The necrotic areas were reduced 2 days later, and were resolved in 1 month. The final visual acuity in his left eye was 20/20 after implantation of an intraocular lens. This case is the first report of ARN after penetrating injury and an intraocular foreign body. ARN may develop after open-globe injury. Keywords: necrotizing herpetic retinopathy, acute retinal necrosis, intraocular foreign body

\section{Purpose}

Acute retinal necrosis (ARN) syndrome can progress rapidly with vision loss due to macular involvement, retinal detachment, or optic neuropathy. ${ }^{1}$ The herpes virus family has been shown to cause ARN syndrome. ${ }^{2}$ According to the criteria, the diagnosis of ARN syndrome should be generally based on clinical appearance and the cause of infection. ${ }^{2}$ Clinical characteristics of ARN include retinal necrosis, location of disease in the periphery, occlusive vasculopathy, and vitreous inflammation. ${ }^{3}$

Although the precise pathogenesis of ARN is not completely understood, it is thought that viruses hide in local ganglion after primary infection, and then they might be reactivated by stimulation such as fever, hormonal changes, ultraviolet exposure, emotional stress, and trauma that could have an influence on immunity. ${ }^{4,5}$

There were a few reported cases of ARN syndrome after periocular trauma, but no case has been reported to be caused by open-globe injury. This is the first report of ARN after penetrating injury by an intraocular foreign body (IOFB).

\section{Case}

A 32-year-old male presented with visual loss after trauma in the left eye. He was hit by iron fragments while he was hammering. A corneal laceration and traumatic cataract were noted. The tip of the IOFB protruded from the surface of the cornea (Figure 1A). Orbital computer tomography showed the IOFB penetrated into the vitreous via the lens (Figure 1B). Visual acuity was hand motion, and the fundus was invisible due to 


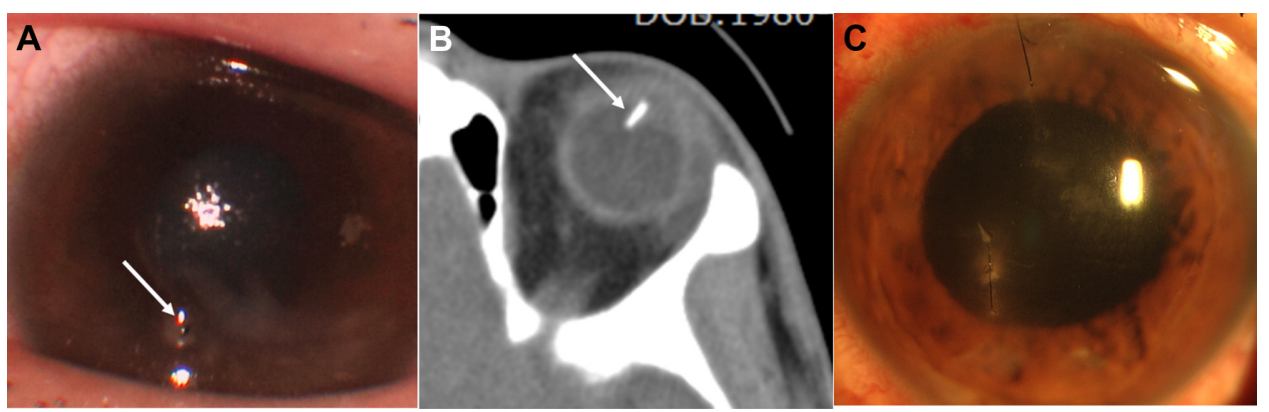

Figure I Preoperative photograph, computer tomography, and postoperative photograph of the left eye. (A) Preoperative photography of left eye shows corneal laceration with corneal edema, the traumatic cataract, and tip of an intraocular foreign body (white arrow). (B) Computer tomography image shows the intraocular foreign body (white arrow) that penetrates into the vitreous via the lens. (C) Postoperative photography on day 3 after the operation shows improved corneal edema.

cataract. On the day he was injured, the IOFB was removed through the corneal laceration. The laceration was closed with 10-0 nylon sutures. Lensectomy and vitrectomy were performed using a $23 \mathrm{G}$ vitrectomy system. Balanced salt solution mixed with vancomycin was infused to prevent the bacterial infection. Ruptured anterior capsule was saved and the lens and posterior capsule were removed by vitreous cutter. During vitrectomy, the retina was confirmed intact with scleral indentation exam. An intraocular lens was not implanted, and the eye remained aphakic. After operation, topical moxifloxacin $0.5 \%$ (Vigamox; Alcon Laboratories, Inc, Fort Worth, TX, USA) was administrated four times a day.

On the first postoperative day, the intraocular inflammation was minimal, and the pupil was well dilated (Figure 1C). The far peripheral retina near the ora serrata was examined by slit-lamp with a 90 diopter lens, and nothing unusual was found. On the second postoperative day, retinal necrosis in the superior far periphery and mild overlying vitreous inflammation were found. On day 3, the necrosis spread circumferentially (Figure 2A-C), and vitreous inflammation (Figure 3A) became more distinct. Hematologic and serologic tests including the Venereal Disease Research Laboratory and fluorescent treponemal antibody absorption tests for syphilis and human immunodeficiency virus were negative, except for immunoglobulin $\mathrm{G}$ (IgG) for herpes simplex virus (HSV). Based on the clinical findings, the patient was presumed to have ARN, and was treated with intravenous acyclovir $800 \mathrm{mg}$ three times per day. Two days later, the necrotic areas of the retina began to reduce.

Acute renal failure occurred as a side effect of acyclovir on the tenth day of administration, and the intravenous acyclovir was discontinued. Renal function was recovered with adequate hydration. The retinal necrosis and vitreous inflammation was resolved completely after 1 month (Figure 3B). A secondary intraocular lens was implanted in the sulcus at 3 months. At the final postoperative visit at 6 months, visual acuity in his left eye was 20/20, and there was no sign of retinitis in both eyes.

\section{Discussion}

The diagnostic criteria of ARN developed by the American Uveitis Society include one or more foci of well-demarcated areas of peripheral retinal necrosis with rapid circumferential progression in the absence of treatment and with evidence of occlusive vasculopathy associated with prominent inflammation

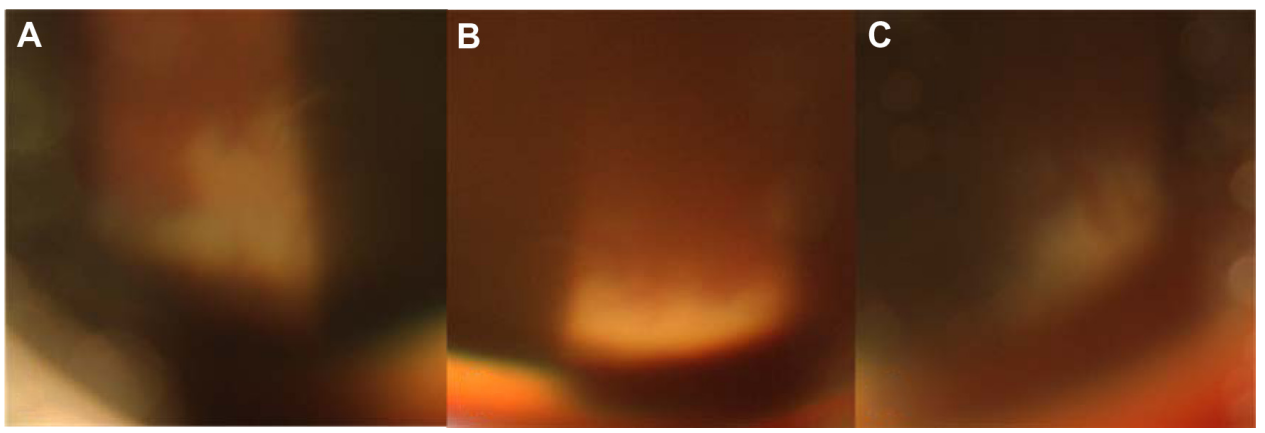

Figure 2 Slit-lamp photography of the superior retina by examination with a 90 diopter Volk lens. (A-C) Slit-lamp photography of the superior retina by examination with a 90 diopter Volk lens shows retinal necrosis spreading circumferentially in the periphery.

Note: The fundus looks blurry due to corneal edema and vitreous haziness. 

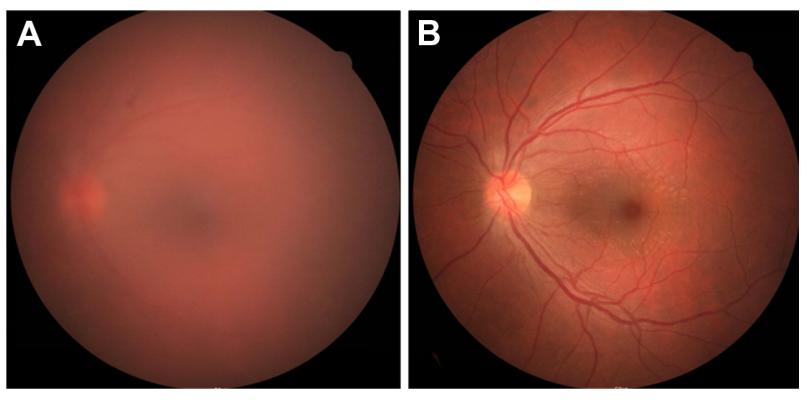

Figure 3 Fundus photography 3 days and I month after the operation. (A) Three days after the operation, fundus photography shows vitreous haziness due to inflammation. (B) Inflammation was resolved at I month after the operation.

in both the vitreous and the anterior chamber. ${ }^{5}$ Virological investigation such as polymerase chain reaction is helpful for the early diagnosis and identification of the causative virus. ${ }^{2}$

In the case presented above, the pattern of retinal necrosis and inflammation corresponded to the criteria, but occlusive vasculopathy was not prominent, possibly due to treatment in the early stage. Although the causative organism was not identified, it is not mandatory in order to make a diagnosis of ARN.

ARN syndrome should be distinguished from a few diseases that present with yellow-white retinal lesion. The manifestations were different from commotio retinae, as there were no abnormal findings of the retina during operation, and given that the white lesion spread rapidly. Necrosis and inflammation had improved by antiviral treatment without steroid. In this regard, ocular sarcoidosis, Behcet's disease and intermediate uveitis could be ruled out. Because the patient's immunity was normal, the disease was less likely to be progressive outer retinal necrosis or cytomegalovirus retinitis. The serologic test for syphilis was negative. As retinal necrosis with inflammation developed after removing the IOFB, it would not be caused by the metal or directly by the trauma. Although microbial culture was not performed, the peripheral circumferential spread of fullthickness retinal necrosis was a typical pattern of ARN.

Although the exact pathogenesis of ARN is still unknown, the viral etiology is now well established with the isolation of viral DNA. Moreover, it is thought that viruses hide in a latent source such as in local ganglion after primary infection, and then they might be reactivated and spread along axonal transport. ${ }^{6,7}$ Once the virus reaches the ganglion cell layer of the retina, the retina is destroyed by the viruses themselves and immune-mediated mechanisms. ${ }^{6,7}$

A variety of stimuli have been shown to trigger the reactivation of latent herpes infection in animal studies, including emotional stress, ultraviolet irradiation, electrical shock, anaphylaxis, pneumococcal infection, and injection of HSV antigens into the corneal stroma. ${ }^{8}$ Cushing et $\mathrm{al}^{9}$ observed that transection of the trigeminal ganglion resulted in recurrence of herpetic disease involving the corresponding ganglion in up to $90 \%$ of subjects. Additionally HSV encephalitis recurred after mild closed head injury and after craniotomy for excision of a meningioma. ${ }^{10}$

Tran et $\mathrm{al}^{11}$ reported a case of ARN after periocular trauma. The patient suffered a head injury 1 week before the ARN syndrome appeared, but his eye was not injured directly. ${ }^{11}$ Thompson et $\mathrm{al}^{5}$ reported three cases of ARN after trauma including periocular skin laceration, corneal abrasion, and after being accidentally struck in the malar area; 1 to 3 weeks later, signs of ARN appeared. In one of the three cases, HSV IgG was positive in the anterior humor and in the other two cases HSV IgG was positive in serum. ${ }^{4}$ Svozílková et al ${ }^{12}$ reported a case of ARN after ocular trauma caused by a finger during a football match. Polymerase chain reaction of the undiluted vitreous of both eyes was positive for varicella zoster virus.

There were a few reported cases of ARN syndrome after periocular trauma, but no case after penetrating injury. This is the first report of ARN after penetrating injury by IOFB. Toh and Borthwick ${ }^{13}$ reported a case of ARN post intravitreal injection of triamcinolone acetonide. Because ARN was observed 6 months after the injection, the trigger factor of ARN might be the steroid, not the trauma associated with the injection.

In this case, daily workup during the hospitalization after removal of the IOFB resulted in early detection and treatment. ARN may develop after open-globe injury as well as after periocular trauma. Being aware of this would be helpful in making a decision for early intervention and would lead to better treatment outcomes.

\section{Disclosure}

The authors report no conflicts of interest in this work.

\section{References}

1. Fisher JP, Lewis ML, Blumenkranz M, et al. The acute retinal necrosis syndrome. Part 1: Clinical manifestations. Ophthalmology. 1982;89(12): 1309-1316.

2. Usui $Y$, Goto H. Overview and diagnosis of acute retinal necrosis syndrome. Semin Ophthalmol. 2008;23(4):275-283.

3. Holland GN. Standard diagnostic criteria for the acute retinal necrosis syndrome. Executive Committee of the American Uveitis Society. Am J Ophthalmol. 1994;117(5):663-667.

4. Vandercam T, Hintzen RQ, de Boer JH, Van der Lelij A. Herpetic encephalitis is a risk factor for acute retinal necrosis. Neurology. 2008;71(16):1268-1274. 
5. Thompson WS, Culbertson WW, Smiddy WE, Robertson JE, Rosenbaum JT. Acute retinal necrosis caused by reactivation of herpes simplex virus type 2. Am J Ophthalmol. 1994;118(2):205-211.

6. Margolis TP, Atherton SS. Herpes simplex virus diseases: posterior segment of the eye. In: Pepose JS, Holland GN, Wilhelmus KR, editors. Ocular Infection and Immunity. St Louis, MO: Mosby; 1996.

7. Atherton SS. Acute retinal necrosis: insights into pathogenesis from the mouse model. Herpes. 2001;8(3):69-73.

8. Green MT, Denkel EC. Herpes simplex virus infections. Latency and reactivation. In: Darrell RW. Viral Diseases of the Eye. Philadelphia, PA: Lea and Febiger; 1985:9-29.

9. Cushing H. Surgical aspects of major neuralgia of trigeminal nerve. Report of 20 cases of operation upon the Gasserian ganglion with anatomical and physiological notes on the consequences of its removal. JAMA. 1905;13:1002-1008.
10. Pazin GJ, Ho M, Jannetta PJ. Reactivation of the herpes simplex virus after decompression of the trigeminal nerve root. $J$ Infect Dis. 1978;138(3):405-409.

11. Tran TH, Stanescu D, Caspers-Velu L, et al. Clinical characteristics of acute HSV-2 retinal necrosis. Am J Ophthalmol. 2004;137(5): 872-879.

12. Svozílková P, Ríhová E, Diblík P, Kuthan P, Kovarik Z, Kalvodová B. Varicella zoster virus acute retinal necrosis following eye contusion: case report. Virol J. 2005;2:77.

13. Toh T, Borthwick JH. Acute retinal necrosis post intravitreal injection of triamcinolone acetonide. Clin Experiment Ophthalmol. 2006;34(4): $380-382$.
Clinical Ophthalmology

\section{Publish your work in this journal}

Clinical Ophthalmology is an international, peer-reviewed journal covering all subspecialties within ophthalmology. Key topics include: Optometry; Visual science; Pharmacology and drug therapy in eye diseases; Basic Sciences; Primary and Secondary eye care; Patient Safety and Quality of Care Improvements. This journal is indexed on Submit your manuscript here: http://www.dovepress.com/clinical-ophthalmology-journal

\section{Dovepress}

PubMed Central and CAS, and is the official journal of The Society of Clinical Ophthalmology (SCO). The manuscript management system is completely online and includes a very quick and fair peer-review system, which is all easy to use. Visit http://www.dovepress.com/ testimonials.php to read real quotes from published authors. 